\title{
p53 N-terminal binding and stabilisation by PIAS3 inhibits MDM2-induced p53 ubiquitination and regulates cell growth
}

\author{
ZIYI ZHAO, LAN WU, HUIMIN SHI and CHUANFANG WU \\ Key Laboratory of Bioresources and Ecoenvironment, Ministry of Education, College of Life Sciences, \\ Sichuan University, Chengdu, Sichuan 610064, P.R. China
}

Received December 13, 2012; Accepted February 17, 2014

DOI: $10.3892 / \mathrm{mmr} .2014 .1993$

\begin{abstract}
Mouse double minute 2 homolog (MDM2) binds to p53 through the 1-52 amino acid region of p53, in order to ubiquitinate p53. MDM2 thus destabilises p53 and inhibits the effect of p53 on cell cycle arrest and apoptosis. In the present study, the 1-52 amino acid region of the wild-type (wt) p53 protein was stably expressed in H1299 cells. The lysate of the transfected cells was then analysed using co-immunoprecipitation. A specific fraction of the precipitate was subjected to mass spectrometry analysis, which revealed that p53 bound to protein inhibitor of activated STAT3 (PIAS3). To the best of our knowledge, the present study is the first to report that the interaction of PIAS3 with p53 occurs through the 1-52 amino acid region of $\mathrm{p} 53$. Overexpression of PIAS3 in the A549 wt p53-expressing cell line was found to significantly increase the half-life of p53 in the presence of cycloheximide, an inhibitor of protein synthesis. However, PIAS3 overexpression did not affect p53 mRNA levels. Furthermore, PIAS3 overexpression was observed to decrease p53 ubiquitination. Protein-protein interaction analysis revealed that PIAS3 binds to the 1-52 amino acid region of $\mathrm{p} 53$, thus disrupts the formation of the p53-MDM2 complex. In addition, overexpression of PIAS3 in A549 cells was found to enhance the transcription of the p53-transactivated target p21/waf1, due to p53 accumulation, which led to an increase in p53 binding to the p21 gene promoter. These findings indicate that this newly identified p53-PIAS3 interaction through the 1-52 amino acid region of p53, reduces p53-MDM2 complex formation, which not only increases the half-life of p53, but also its transactivation of target genes.
\end{abstract}

Correspondence to: Miss Chuan Fang Wu, Key Laboratory of Bioresources and Ecoenvironment, Ministry of Education, College of Life Sciences, Sichuan University, 29 Wangjiang Road, Chengdu, Sichuan 610064, P.R. China

E-mail: 879413966@qq.com

Key words: p53, mouse double minute 2 homolog, p21, protein inhibitor of activated STAT3, ubiquitination, cell cycle

\section{Introduction}

p53 is a major cell proliferation suppressor with a central role in a complex regulatory network. p53 inhibits the cell cycle through several different mechanisms and is capable of inducing cell death when required (1). These inhibitory functions of p53 on cell growth are important to prevent tumourigenesis and the loss of wild-type p53 has been demonstrated to increase the risk of cancer in humans $(2,3)$. Despite its crucial role in preventing malignant cell growth, p53 is not fundamental to normal cell proliferation and differentiation, with wild-type p53 being absent during embryonic development (4). Additionally, p53 overexpression in normal cells is capable of inducing fatal damage due to its excessive activity. Thus, p53 homeostasis is vital for normal cell development and proliferation.

Mouse double minute 2 homolog (MDM2), which is encoded by the MDM 2 oncogene, modulates the levels of p53 and is involved in a negative feedback loop, which regulates p53 protein expression. Upon phosphorylation, $\mathrm{p} 53$ has a relatively short half-life, as MDM2 binds and ubiquitinates the phoshphorylated form of $\mathrm{p} 53$, which is then ultimately degraded (5). Under pathological conditions, the half-life of p53 is prolonged, due to compromised MDM2-induced ubiquitination as a result of the activation of multiple inhibitory pathways, which in certain cases cause p53 to become hyperactive (6). In addition, numerous p53 target genes regulate the trans-activity of MDM2. p53-MDM2 feedback is thus important when cells are challenged by factors that may stimulate development and growth processes. MDM2 has been found to interact with $\mathrm{p} 53$ through three major mechanisms: (i) Through binding to the $\mathrm{N}$-terminus of p53 to inhibit its function as a transcription factor (7,8); (ii) through acting as an E3 ubiquitin ligase and promoting the ubiquitination and degradation of p53 (9) and (iii) through facilitating p53 translocation from the nucleus to the cytoplasm in order to separate p53 from regulatory sites within its target genes.

Interruption of the MDM2-p53 feedback loop impacts cell survival. p14ARF is a tumour suppressor involved in interrupting this feedback loop (10-12). It binds to MDM2, retaining MDM2 in the nucleus and separating it from p53 $(13,14)$, thus impeding the formation of the MDM2-p53 complex and disrupting the feedback loop. Another protein that interrupts the MDM2-p53 feedback loop is MDMx; an MDM2 homologue. MDMx competes with p53 for ubiquitination by MDM2, ultimately resulting in the degradation of MDMx instead of p53 (15). 
The present study investigated approaches to screen for proteins that compete with p53 for binding to MDM2. Co-immunoprecipitation (Co-IP) was performed using the 1-52 amino acid region of p53 as bait. The mechanism underlying the effect of PIAS3 on the MDM2-p53 negative feedback loop, which differs from the manner in which p53 accumulates under conditions of DNA damage and proteinase inhibitor treatment, was also investigated. Understanding the mechanism by which PIAS3 affects the MDM2-p53 feedback loop may provide novel insight for tumour suppression by increasing the stability of the p53 protein.

\section{Materials and methods}

Cell lines and plasmids. Human HEK293, H1299 and A549 cells (American Type Culture Collection, Manassas, VA, USA) were grown in Dulbecco's modified Eagle's medium (DMEM) supplemented with $10 \%$ (v/v) foetal bovine serum (FBS), $100 \mathrm{U} / \mathrm{ml}$ penicillin and $100 \mu \mathrm{g} / \mathrm{ml}$ streptomycin at $37^{\circ} \mathrm{C}$, in $5 \% \mathrm{CO}_{2}$. To generate truncated p53 N-terminal proteins and full-length p53 proteins fused to $\mathrm{FLAG}^{\circledR}$ tags (Sigma-Aldrich, St. Louis, MO, USA) at the N-terminus, reverse transcription-polymerase chain reaction (RT-PCR) was performed using the following primer sequences: P1, 5'-GGAATTCCATATGTACCCATACGATGTTCCAGATTA CGCTGAGGAGCCGCAGTCAGA-3' and either P2, 5'-CCG CTCGAGTTACCTGCTCCCCCCTGGCTCCTT-3', or P3, 5'-CCGCTCGAGGATTACAAGGATGACGACGATAAG-3' (BGI, Beijing, China). The respective PCR products were cloned into the pcDNA3.1 vector using the NheI and XhoI restriction sites. To generate truncated $\mathrm{p} 53 \mathrm{~N}$-terminal proteins and full-length p53 proteins fused to haemagglutinin (HA) tags at the N-terminus (Life Technologies, San Diego, CA, USA), RT-PCR was performed using the following primer sequences: P4, 5'-GGAATTCCATATGTACCCATACGATG TTCCAGATTACGCTGAGGAGCCGCAGTCAGA-3' and either P5, 5'-CCGCTCGAGAGCCCTGCTCCCCCCTGGC TCC-3', or P6, 5'-CCGCTCGAGTCAGTCTGAGTCAGGCCC TTCTGT-3' (BGI). The respective PCR products were then cloned into the pcDNA3.1 vector using the NheI and XhoI restriction sites.

Antibodies. The mouse monoclonal anti-FLAG and -HA antibodies (Abcam PLC, Cambridge, MA, USA) were used for western blot analysis and the detection of the IP products of the Flag- and HA-tagged proteins, respectively. The mouse monoclonal DO-1 anti-p53 antibody (Santa Cruz Biotechnology, Inc., Santa Cruz, CA) was used for the detection of ubiquitinated p53 and p53 immunoprecipitates. The mouse monoclonal anti-p21 antibody (Abcam PLC) was used for the detection of endogenous p21 protein. The mouse monoclonal anti-MDM2 and -PIAS3 antibodies (Abcam PLC) were used for the detection and immunoprecipitation of endogenous MDM2 and PIAS3 proteins, respectively.

Quantitative PCR. Quantitative PCR was performed on the iCycler instrument (Bio-Rad) as follows: $95^{\circ} \mathrm{C}$ for $5 \mathrm{~min}, 40$ cycles $\left(95^{\circ} \mathrm{C}\right.$ for $30 \mathrm{sec}, 60^{\circ} \mathrm{C}$ for $30 \mathrm{sec}$ and $72^{\circ} \mathrm{C}$ for $20 \mathrm{sec}$ ). Data were exported from iCycler software (Bio-Rad) and imported into Excel (Microsoft, Redmond, WA, USA).
Western blot analysis. Cells were harvested and protein concentrations were quantified using a Bio-Rad protein assay kit (Bio-Rad, Hercules, CA, USA). Protein samples were then subjected to sodium dodecyl sulfate-polyacrylamide gel electrophoresis (SDS-PAGE) and transferred to Immunobilon-P polyvinylidene fluoride membranes (Millipore, Billerica, MA, USA) using a Semi-Dry Transfer kit (Bio-Rad). Membranes were blocked using $10 \%$ (w/v) non-fat dry milk and subsequently incubated in the aforementioned primary antibodies and rabbit polyclonal horseradish peroxidase-labeled secondary antibodies to mouse IgG (Abcam PLC). Immunoreactive signals were visualised using enhanced chemiluminescence (Amersham Pharmacia Biotech, Little Chalfont, UK) and exposed to X-ray film.

Co-IP assay. Co-IP of endogenously expressed proteins was performed using HEK293 cells transfected with pcDNA3.1-Flag-p53NT, pcDNA3.1-HA-PIAS3 or empty vector. After $24 \mathrm{~h}$, cells were harvested using 1\% NP40 lysis buffer. Lysates were then incubated overnight with $2.5 \mu \mathrm{g}$ anti-Flag antibody and Protein G beads using a Protein G Immunoprecipitation kit (Sigma-Aldrich). The resulting complexes were washed, denatured and eluted according to the manufacturer's instructions.

Silver staining of SDS-polyacrylamide gels. Following electrophoresis, the SDS-polyacrylamide gels were soaked in fixative containing $25 \%$ ethanol and $10 \%$ acetic acid for $30 \mathrm{~min}$. Gels were then transferred to conditioning medium containing $0.4 \mathrm{M}$ sodium acetate ( $\mathrm{pH}$ 6.0), 30\% ethanol, $4.4 \mathrm{mM}$ sodium thiosulfate and $1 \%$ glutaraldehyde for $20 \mathrm{~min}$. Following conditioning, gels underwent at least three 5 min washes in $\mathrm{ddH}_{2} \mathrm{O}$. Each gel was then soaked in silver nitrate solution $(0.1 \%$ silver nitrate and $0.01 \%$ formaldehyde) for $20 \mathrm{~min}$, followed by development using developer solution $(2.5 \%$ sodium carbonate and $0.015 \%$ formaldehyde). Development was terminated with the addition of acetic acid.

Proliferation assay. Cell proliferation was assayed using the phenazine methosulfate and 3-(4,5-dimethylthiazol-2-yl)5-(3-carboxymethoxyphenyl)-2-(4-sulfophenyl)-2H-tetrazolium (MTS)-based Cell Titer $96{ }^{\circledR}$ AQueous One Solution Cell Proliferation assay (Promega Corporation, Madison, WI, USA). Approximately 1000 cells/well were plated onto 96-well plates. After $24 \mathrm{~h}$, the MTS mix was added at a final volume of $20 \mu \mathrm{l} / 100 \mu \mathrm{l}$ medium and incubated for an additional $60 \mathrm{~min}$ under the same conditions. Following the addition of $10 \%$ SDS, the absorbance of formazan was measured at $490 \mathrm{~nm}$ using an Evolution 60S (Thermo Scientific, Waltham, MA, USA). All assays were performed in triplicate every other day for 10 days. The results are expressed as the mean \pm standard error.

Soft agar assay. Efficiency of growth in soft agarose was determined by plating the cells in DMEM containing $0.33 \%$ agarose (Indubiose A37; IBF, Villeneuve-la-Garenne, France) with $10 \%$ FBS above a layer of $0.5 \%$ agarose in the same culture medium. Colonies were counted two weeks subsequent to plating. Cultures were examined using an inverted microscope (Leica DM6000 CS; Leica, Hong Kong, China). 


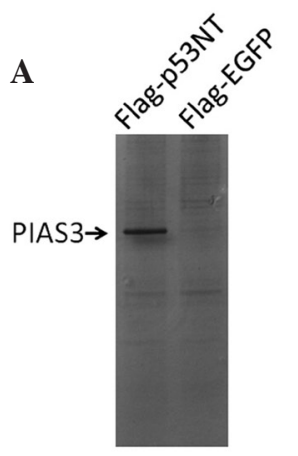

B

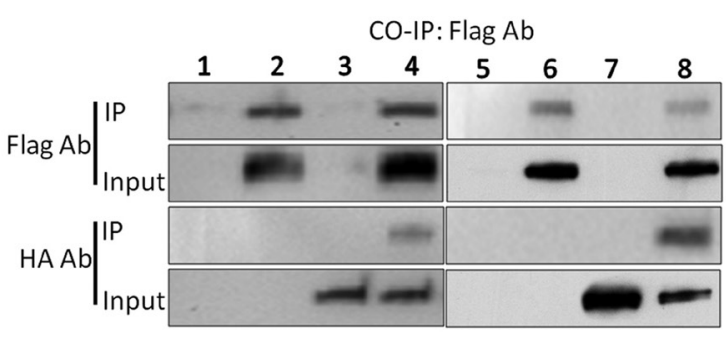

C

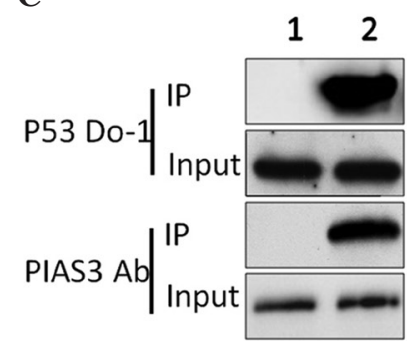

Figure 1 Identification of a novel p53 binding protein. (A) Identification of a novel p53 binding protein using silver staining. (B) Co-IP analysis of protein-protein interactions between p53NT and PIAS3. PcDNA3.1-Flag-p53NT and pcDNA3.1-HA-PIAS3 were transfected into HEK293 together or independently. After $24 \mathrm{~h}$, Co-IPs were performed using an anti-Flag antibody and the inputs and IP products were assayed using anti-Flag or -HA antibodies, respectively. Lanes 1 and 5, untreated HEK293 cells; lane 2, HEK293 cells transfected with pcDNA3.1-Flag-p53NT; lanes 3 and 7, HEK293 cells transfected with pcDNA3.1-HA-PIAS3; lane 4, HEK293 cells co-transfected with pcDNA3.1-Flag-p53NT and pcDNA3.1-HA-PIAS3; lane 6, HEK293 cells transfected with pcDNA3.1-Flag-p53 and lane 8, HEK293 cells co-transfected with pcDNA3.1-Flag-p53 and pcDNA3.1-HA-PIAS3. (C) Co-IP analysis of the endogenous interaction between p53 and PIAS3. Untransfected HEK293 cells were lysed and Co-IP analyses were performed using the anti-p53 antibody DO-1 (lane 1) or rabbit immunoglobulin G (lane 2) as negative control. PIAS3, protein inhibitor of activated STAT3; p53NT, p53 N-terminus; HA, haemagglutinin; IP, immunoprecipitation; EGFP, enhanced green fluorescent protein.

Tandem mass spectrometry (MS/MS) identification of novel p53 binding proteins. MS/MS identification of phosphopeptides was conducted using a 4800 MALDI TOF/TOF ${ }^{\mathrm{TM}}$ analyser (Applied Biosystems, Carlsbad, CA, USA). Tryptic digests of samples were re-dissolved in $0.1 \%$ trifluoroacetic acid and $50 \%$ acetonitrile, then mixed 1:1 with a matrix consisting of $20 \mathrm{mg} / \mathrm{ml}$ DHCA, $50 \%$ acetonitrile and $5 \mathrm{mM} \mathrm{NH}_{4} \mathrm{HPO}_{4}$, prior to spotting on a sample plate. The sample plate was externally calibrated using a Mass Standards kit (Applied Biosystems). The peptide MALDI-TOF/TOF spectra were acquired manually by inputting the peptide mass into the precursor mass window. MS/MS spectra were collected using $\geq 2,000$ laser shots and were searched against a Gossypium peptide sequence database annotated from a Gossypium expressed sequence tag (EST) database (downloaded from http://www.ncbi.nlm.nih.gov; release date December 9 2010; including 507,959 EST sequences and 62,267,048 residues) using MASCOT 2.2 software (Matrix Science, Oxford, UK) with the following settings: Enzyme, trypsin; MS tolerance, $0.3 \mathrm{Da}$; MS/MS tolerance, $0.5 \mathrm{Da}$; maximum number of missed cleavages, 1 ; peptide charge, $1^{+}$; fixed modifications, carbamidomethylation of Cys; variable modifications, oxidation of Met, phosphorylation and sulphation of Ser, Thr and Tyr. Peptides with an ion score $>15$ were considered to possess significant homology $(\mathrm{P}<0.1)$ rather than being a random event. Analyses of spectra were then performed by hand to further validate the identification of these peptides.

Statistical analysis. All results are presented as the average \pm standard error of the mean. Student's t-test was used for comparison between two groups. The difference is considered as statistically significant when $\mathrm{P}<0.05$.

\section{Results}

Selection and identification of a novel p53 binding protein. pcDNA3.1-Flag-p53NT was constructed and was stably transfected into H1299 cells along with the pcDNA3.1 empty vector. H1299 cell lysates were then subjected to immunoprecipitation using an anti-FLAG antibody. The products of the Co-IP assay were separated using 10\% SDS-PAGE and silver stained. Numerous protein bands were observed on the pcDNA3.1-Flag-p53NT-transfected H1299 cell gel compared with the empty vector gel (Fig. 1A). The bands were excised from the gel and the proteins were recovered and sent for MS analysis (Beckman Coulter, Inc., Brea, CA, USA). MS revealed sequences of several types of protein. The most significant signal was from the PIAS3 protein. The corresponding co-IP band of PIAS3 was also the strongest; therefore, PIAS3 was selected to be the focus of this study. To assess the interaction between p53 and PIAS3, HEK293 cells were transfected with either pcDNA3.1-FLAG-p53NT and pcDNA3.1-HA-PIAS3 or pcDNA3.1-FLAG-p53 and pcDNA3.1-HA-PIAS3. Each group of transfected cells was lysed $24 \mathrm{~h}$ after transfection and the lysates were subjected to Co-IP. Western blot analysis using antibodies corresponding to the Co-IP products and inputs, revealed that PIAS3 binds to the truncated p53 NT protein and the wild-type p53 protein in vitro (Fig. 1B). To further assess the interaction between endogenous p53 and PIAS3, untransfected HEK293 cells were analysed using Co-IP. Similar results were observed, showing that endogenous PIAS3 also binds to p53 (Fig. 1C).

PIAS3 increases the stability and transactivity of p53 by inhibiting its ubiquitination. In the present study, PIAS3 was observed to bind to 533 through the 1-52 amino acid region of p53 (Fig. 1B). This region was previously reported to be the domain through which p53 binds to MDM2 (16). The p53-MDM2 interaction leads to the ubiquitination of p53, resulting in $\mathrm{p} 53$ degradation (17). Therefore, the present study aimed to investigate whether PIAS3 affects p53 protein stability. 553 protein expression was assessed in HEK293 cells 12, 24, 36 and $48 \mathrm{~h}$ after transient transfection with pcDNA3.1-PIAS3 or with the empty vector. Western blot analysis revealed a significant time-dependent increase in p53 protein expression following transfection, showing that PIAS3 is capable of increasing p53 protein levels (Fig. 2A). However, no significant difference was observed in p53 mRNA expression following transfection in these cells (Fig. 2B). HEK293 cells overexpressing PIAS3 and control HEK293 cells were then treated with cycloheximide, 
A

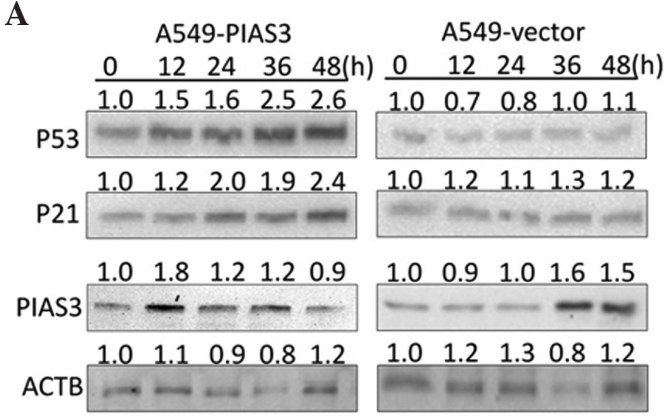

C

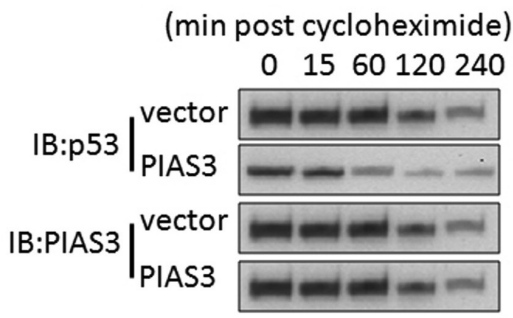

$\mathbf{E}$

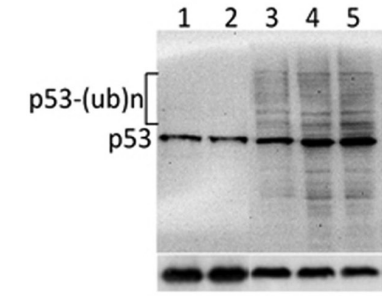

B

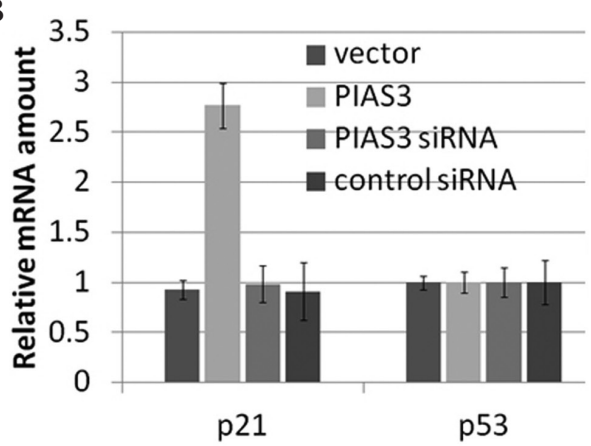

D

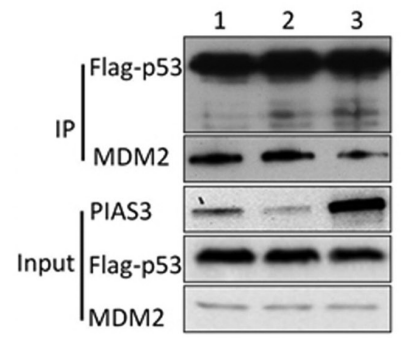

$\mathbf{F}$

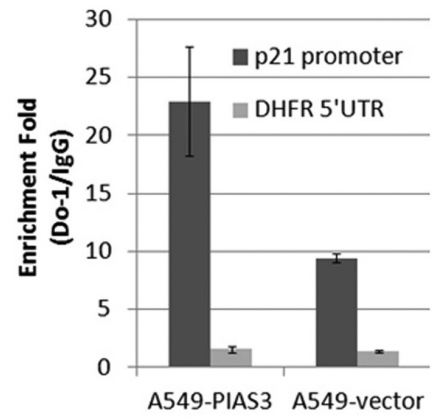

Figure 2. PIAS3 inhibits the ubiquitination and increases the transactivity of p53. (A) Overexpression of PIAS3 increased p53 protein expression without altering p53 mRNA levels. (B) Overexpression of PIAS3 was not found to change p21 mRNA expression. A549 cells were stably transfected with PcDNA3.1-PIAS3 and p53 and p21 mRNA levels were detected using quantitative polymerase chain reaction analysis. (C) Overexpression of PIAS3 increases the half-life of p53. (D) Competitive binding of PIAS3 to p53 inhibits p53-MDM2 binding. HEK293 cells were co-transfected with: lane 1, Flag-p53 and MDM2; lane 2, Flag-p53, MDM2 and the empty vector and lane 3, Flag-p53, MDM2 and PIAS3. (E) Overexpression of PIAS3 inhibits MDM2-mediated p53 ubiquitination. Lane 1, A549-PIAS3; lane 2, A549-PIAS3 incubated with ALLN; lane 3, A549 incubated with ALLN; lane 4, A549-empty vector incubated with ALLN and lane 5, A549-pcDNA31-Flag incubated with ALLN. (F) Overexpression of PIAS3 was observed to promote the binding activity of p53 to the p21 promoter. Chromatin IP using an anti-p53 antibody followed by qPCR analysis was performed on the p21 promoter. PIAS3, protein inhibitor of activated STAT3; IP, immunoprecipitation; siRNA, small interfering RNA; DFHR, dihydrofolate reductase; UTR, untranslated region; ACTB, actin beta; MDM2, mouse double minute 2 homolog.

a translational elongation inhibitor, and cell lysates were harvested to measure the half-life of p53 (18). Western blot analysis revealed that cycloheximide attenuated p53 degradation in the HEK293 cells overexpressing PIAS3, but not in the HEK293 cells transfected with the empty pcDNA3.1 vector (Fig. 2C). In vitro Co-IP assay revealed that overexpression of PIAS3 competitively inhibited p53-MDM2 binding through binding to the p53 protein (Fig. 2D). These findings suggest that PIAS3 increases p53 expression in a post-translational manner and strongly suggests the involvement of PIAS3 in disrupting the formation of the p53-MDM2 complex and inhibiting MDM2-induced p53 ubiquitination (Fig. 2E). p53 is an important transcription factor involved in a complex regulatory network; therefore, its intracellular stability is critical for the normal functioning of its downstream targets, including p21 (19). Thus, the effect of PIAS3 on p21 expression was investigated. Chromatin IP analysis of HEK 293 cells transiently transfected with pcDNA3.1-PIAS3 revealed that the PIAS3 overexpression-induced p53 accumulation increased the binding of p53 to its responsive elements in DNA clusters in the $\mathrm{p} 21$ promoter region (Fig. 2F). In addition, quantitative PCR and western blot analyses showed that $\mathrm{p} 21$ expression was upregulated at the mRNA and protein levels in the PIAS3 overexpressing cells, while no difference was detected in the HEK293 cells transfected with the empty pcDNA3.1 vector (Fig. 2A and B).

PIAS3 overexpression inhibits cell growth rate and tumour formation in a p53-dependent manner. It is well established that p53 overexpression enhances the induction of apoptosis and cell cycle arrest. In the present study, PIAS3 was observed to stabilise the p53 protein through disrupting the formation of the p53-MDM2 complex; therefore, the effect of PIAS3 on cell proliferation was investigated. To assess the effect of PIAS3 on cell proliferation, A549 cells were stably transfected with pcDNA3.1-Flag-PIAS3 or the empty pcDNA3.1 vector and cell growth curves were constructed. In order to determine whether PIAS3 overexpression induces cell cycle arrest in a 
A

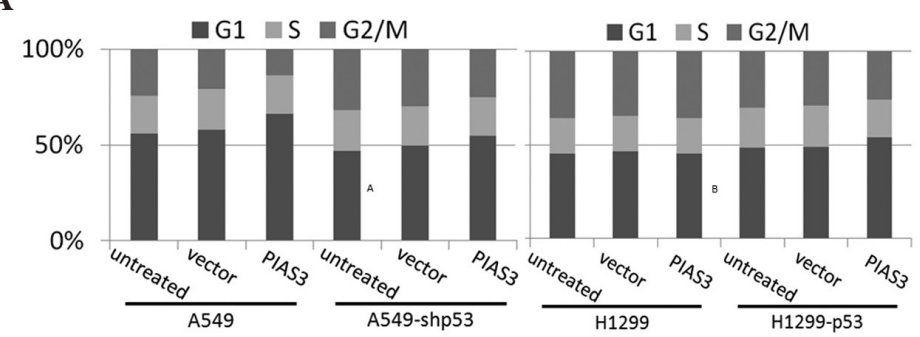

B

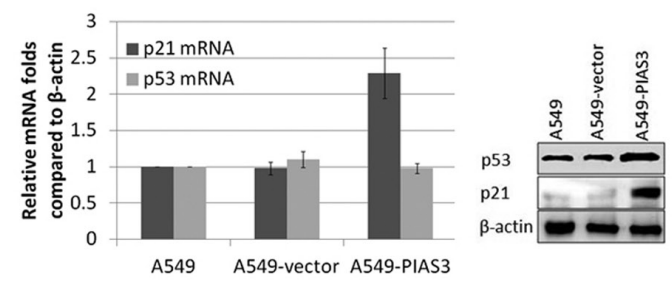

C

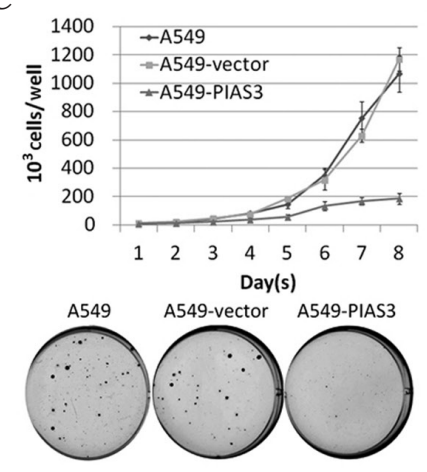

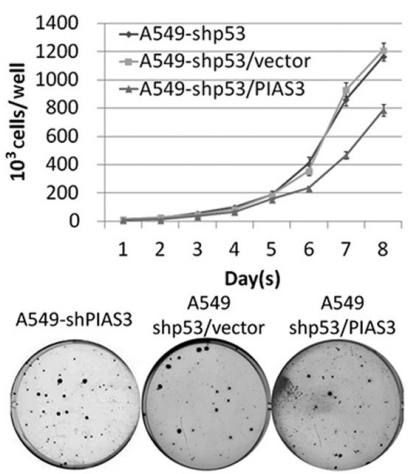
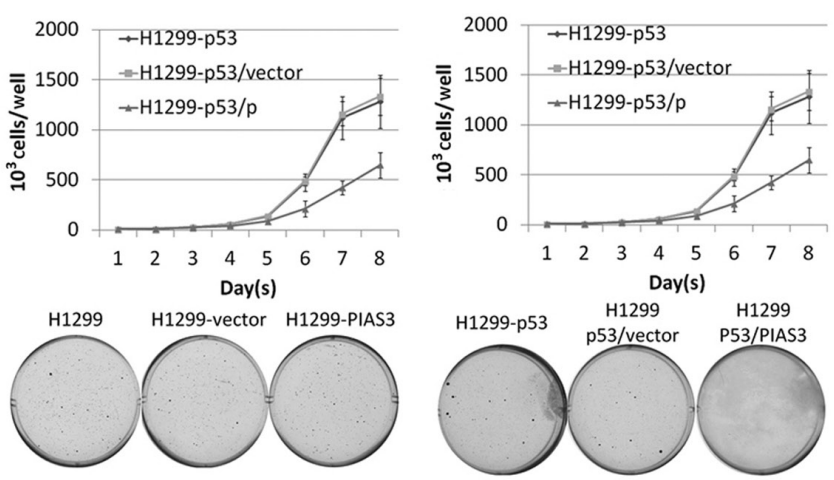

Figure 3. P53-induced cell proliferation and colony formation in soft agar are regulated by PIAS3. (A and B) Overexpression of PIAS3 affects the cell cycle in a manner dependent on p53-induced p21 transcription. (C) Overexpression of PIAS3 increases p53-induced supression of cell proliferation and colony formation. PIAS3, protein inhibitor of activated STAT3.

p53-dependent manner, A549 cells stably transfected with pcDNA3.1-Flag-PIAS3 or the empty vector were subjected to flow cytometric analysis subsequent to DNA staining. Overexpression of PIAS3 was found to increase the proportion of cells in $\mathrm{G}_{0} / \mathrm{G}_{1}$-phase (Fig. 3A). By contrast, PIAS3 overexpression had no effect on the cell cycle in the p53-null H1299 cell line or in p53-knockdown A549 cells. The accumulation of p53 was observed to cause cell cycle changes through the upregulation of p21 (Fig. 3B). Furthermore, PIAS3 overexpression was found to decelerate cell growth rate (Fig. 3C). Soft agar assay on A549 cells overexpressing PIAS3 and A549 cells transfected with the empty vector revealed that PIAS3 inhibits the rate of tumour formation. By contrast, PIAS3 overexpression in NCl-H1299 cells, a p53-null cell line, had no effect on cell growth or tumour formation. To further investigate this finding, H1299 cells were transfected with pcDNA3.1-Flag-p53 to generate a stably transfected wild-type p53-expressing cell line, H1299-wtp53. PcDNA5-PIAS3 or the pcDNA5 empty vector were then transfected into H1299-wtp53 cells and stably overexpressing cell lines were obtained. These cell lines were utilised to construct cell growth curves and perform the soft agar assay. Exogenous wt-p53 was found to inhibit cell growth rate and tumour formation through PIAS in the H1299-wtp53 cell line.

\section{Discussion}

Investigation into the p53 network has revealed increasing numbers of proteins that are involved in the MDM2-p53 negative feedback circuit. Such proteins include, p300, E2F1 and the $\mathrm{p} 38$, casein kinase 1 and ataxia telangiectasia mutated kinases (20-23). Notably, these proteins are capable of mediating the function of MDM2 by regulating p53. Phosphorylated
MDM2 loses its capacity to regulate p53, as phosphorylation induces MDM2 self-ubiquitination and subcellular translocation.

Accumulation of the $\mathrm{p} 53$ protein is vital for $\mathrm{p} 53$ activation; triggering its DNA binding, transcriptional regulation and other regulatory functions. Under normal conditions, MDM2 controls the accumulation of p53 by regulating its half-life and maintains a relatively low level of p53 protein expression $(9,24,25)$. The p53 binding domain in MDM2 binds to a transcriptional regulatory domain at the N-terminus of $\mathrm{p} 53$. Upon p53-MDM2 binding, p53 is phosphorylated by the RING domain of MDM2 (26). Ubiquitinated p53 is then translocated to the cytoplasm or the nucleus and is ultimately degraded by 26s proteasomes $(27,28)$. Furthermore, p53 regulates MDM2 transcription, forming an automatic negative feedback loop together with MDM2-mediated p53 degradation (29). Thus, the MDM2-p53 feedback loop is an important mechanism, which must be overcome in order for p53 to become activated. Upon DNA damage, the p53 protein is highly phosphorylated by a series of kinases, including ATM/ataxia telangiectasia and Rad3 related and checkpoint kinase 2 (30). Previous reports have demonstrated that $\mathrm{p} 53$ phosphorylation at Ser20 promotes p53 stability and activates the DNA repair process (31-33). The p53-MDM2 interaction is essential to the ubiquitination and degradation of $\mathrm{p} 53$; therefore, mechanisms blocking the formation of the p53-MDM2 feedback loop enhance p53 stability.

PIAS3 is an E3 small ubiquitin-like modifier (SUMO)-protein ligase, which belongs to the PIAS family and covalently attaches SUMO proteins to specific target substrates. SUMO modification of p53 modulates p53 transcriptional activity (34) and PIAS3-mediated heterogeneous nuclear ribonucleoprotein $\mathrm{K}$ (hnRNP-K) SUMOylation has been reported to increase hnRNP-K stability, as well as its interaction with p53 and p21, 
leading to cell cycle arrest (35). Notably, in the present study, PIAS3 was observed to bind directly to the $\mathrm{N}$ terminus of p53. In addition, the interaction between PIAS3 and P53 was found to increase the stability of p53 and stimulate p53-induced p21 transcription, which may lead to cell cycle arrest (data not shown).

The interaction between MDM2 and p53 is a highly regulated process. MDM2 binds directly to the first transactivation domain (amino acids, 20-40) of p53, inhibiting its capacity to interact with transcriptional co-activators (36). However, it has also been shown that the p53 C-terminal domain interacts directly with the MDM2 N-terminus (37). C-terminal modification of p53 has been reported to induce full dissociation of MDM2-p53 complexes in cells. Thus, the N-terminus, as well as the $\mathrm{C}$-terminus bind to the $\mathrm{N}$-terminus of MDM2. Further research on the interaction between the C-terminus of p53 and PIAS3 is required.

In the present study, a novel p53 binding protein, PIAS3, was identified and binding of PIAS3 to p53 was found to activate the transactivity of p53. Two different approaches were used to analyse the effect of PIAS3 on p53 transactivation. Chromatin IP assays were performed to demonstrate the increased DNA binding activity of p53 in PIAS3 overexpressing cells. In addition, the expression of the p53 target gene p21 was detected using western blot analysis and indicated that PIAS3 induced functional activation of the p53 protein. Furthermore, the overexpression of PIAS3 was found to inhibit MDM2-induced p53 ubiquitination and increase the half-life of p53. In addition, DNA binding and the transactivation of the $\mathrm{p} 21 /$ waf1 promoter were induced maximally when the stabilised p53 levels had returned to control levels.

\section{References}

1. Shaper NJ, Harrison M and Bates T: Impact of laparoscopic cholecystectomy on surgical training. Ann R Coll Surg Engl 78: $39-42,1996$

2. Evans SC and Lozano G: The Li-Fraumeni syndrome: an inherited susceptibility to cancer. Mol Med Today 3: 390-395, 1997.

3. Attardi LD and Jacks T: The role of p53 in tumour suppression: lessons from mouse models. Cell Mol Life Sci 55: 48-63, 1999.

4. Choi J and Donehower LA: p53 in embryonic development: maintaining a fine balance. Cell Mol Life Sci 55: 38-47, 1999.

5. Piette J, Neel H and Maréchal V: Mdm2: keeping p53 under control. Oncogene 15: 1001-1010, 1997.

6. Karanicolas J and Brooks CL III: The structural basis for biphasic kinetics in the folding of the WW domain from a forming-binding protein: Lessons for protein design? Proc Natl Acad Sci USA 100: 3954-3959, 2003.

7. Kussie PH, Gorina S, Marechal V, Elenbaas B, Moreau J, Levine AJ and Pavletich NP: Structure of the MDM2 oncoprotein bound to the $\mathrm{p} 53$ tumor suppressor transactivation domain. Science 274: 948-953, 1996.

8. Momand J, Zambetti GP, Olson DC, George D and Levine AJ: The $\mathrm{mdm}-2$ oncogene product forms a complex with the p53 protein and inhibits p53-mediated transactivation. Cell 69: 1237-1245, 1992.

9. Haupt Y, Maya R, Kazaz A and Oren M: Mdm2 promotes the rapid degradation of p53. Nature 387: 296-299, 1997.

10. Kamijo T, Weber JD, Zambetti G,Zindy F, Roussel MF and Sherr CJ: Functional and physical interactions of the ARF tumor suppressor with p53 and Mdm2. Proc Natl Acad Sci USA 95: 8292-8297, 1998.

11. Pomerantz J, Schreiber-Agus N, Liégeois NJ, Silverman A, Alland L, Chin L, Potes J, Chen K, Orlow I, Lee HW, Cordon-Cardo C and DePinho RA: The Ink4a tumor suppressor gene product, p19Arf, interacts with MDM2 and neutralizes MDM2's inhibition of p53. Cell 92: 713-723, 1998.

12. Honda R and Yasuda H: Association of p19(ARF) with Mdm2 inhibits ubiquitin ligase activity of $\mathrm{Mdm} 2$ for tumor suppressor p53. ЕMBO J 18: 22-27, 1999.
13. Tao W and Levine AJ: P19(ARF) stabilizes p53 by blocking nucleo-cytoplasmic shuttling of Mdm2. Proc Natl Acad Sci USA 96: 6937-6941, 1999.

14. Weber JD, Taylor LJ, Roussel MF, Sherr CJ and Bar-Sagi D: Nucleolar Arf sequesters Mdm2 and activates p53. Nat Cell Biol 1: 20-26, 1999.

15. Shadfan M, Lopez-Pajares V and Yuan ZM: MDM2 and MDMX: Alone and together in regulation of $\mathrm{p} 53$. Transl Cancer Res 1: 88-89, 2012.

16. Chen J, Marechal V and Levine AJ: Mapping of the p53 and mdm-2 interaction domains. Mol Cell Biol 13: 4107-4114, 1993.

17. Momand J, Zambetti GP, Olson DC, George D and Levine AJ: The mdm-2 oncogene product forms a complex with the $\mathrm{p} 53$ protein and inhibits p53-mediated transactivation. Cell 69: 1237-1245, 1992.

18. Takimoto R, Wang W, Dicker DT, Rastinejad F, Lyssikatos J and el-Deiry WS: The mutant p53-conformation modifying drug, CP-31398, can induce apoptosis of human cancer cells and can stabilize wild-type p53 protein. Cancer Biol Ther 1: 47-55, 2002.

19. el-Deiry WS, Tokino T, Velculescu VE, Levy DB, Parsons R, Trent JM, Lin D, Mercer WE, Kinzler KW and Vogelstein B: WAF1, a potential mediator of p53 tumor suppression. Cell 75: 817-825, 1993.

20. Martin K, Trouche K, Hagemeier C, Sørensen TS, La Thangue NB and Kouzarides T: Stimulation of E2F1/DP1 transcriptional activity by MDM2 oncoprotein. Nature 375: 691-694, 1995.

21. Lughran O and La Thangue NB: Apoptotic and growth-promoting activity of E2F modulated by MDM2. Mol Cell Biol 20: 2186-2197, 2000

22. Grossman SR, Perez M, Kung AL, et al: P300/MDM2 complexes participate in Mdm2-mediated p53 degradation. Mol Cell 2: 405-415, 1998

23. Avantaggiati ML, Ogryzko V, Gardner K, Giordano A, Levine AS and Kelly K: Recruitment of p300/CBP in p53-dependent signal pathways. Cell 89: 1175-1184, 1997.

24. Kubbutat MH, Jones SN and Vousden KH: Regulation of p53 stability by Mdm2. Nature 387: 299-303, 1997.

25. Yu ZK, Geyer RK and Maki CG: MDM2-dependent ubiquitination of nuclear and cytoplasmic P53. Oncogen 19: 5892-5897, 2000.

26. Fang S, Jensen JP, Ludwig RL, Vousden KH and Weissman AM: $\mathrm{Mdm} 2$ is a RING finger-dependent ubiquitin protein ligase for itself and p53. J Biol Chem 275: 8945-8951, 2000.

27. Geyer RK, Yu ZK and Maki CG: The MDM2 RING-finger domain is required to promote p53 nuclear export. Nat Cell Biol 2: 569-573, 2000

28. Xirodimas DP, Stephen CW and Lane DP: Compartmentalization of p53 and Mdm2 is a major determinant for Mdm2-mediated degradation of p53. Exp Cell Res 270: 66-77, 2001.

29. Barak Y, Juven T, Haffner R and Oren M: Mdm2 expression is induced by wild type p53 activity. EMBO J 12: 461-468, 1993.

30. Durocher D and Jackson SP: DNA-PK, ATM and ATR as sensors of DNA damage: variations on a theme? Curr Opin Cell Biol 13: 225-231, 2001.

31. Chehab NH, Malikzay A, Stavridi ES and Halazonetis TD: Phosphorylation of Ser-20 mediates stabilization of human p53 in response to DNA damage. Proc Natl Acad Sci USA 96: 13777-13782, 1999.

32. Dumaz N, Milne DM, Jardine LJ and Meek DW: Critical roles for the serine 20 , but not the serine 15 , phosphorylation site and for the polyproline domain in regulating p53 turnover. Biochem J 359: 459-464, 2001.

33. Unger T, Juven-Gershon T, Moallem E, Berger M, Vogt Sionov R, Lozano G, Oren M and Haupt Y: Critical role for Ser20 of human p53 in the negative regulation of p53 by Mdm2. EMBO J 18: 1805-1814, 1999.

34. Stindt MH, Carter S, Vigneron AM, Ryan KM, and Vousden KH: MDM2 promotes SUMO-2/3 modification of p53 to modulate transcriptional activity. Cell Cycle 10: 3176-3188, 2001.

35. Lee SW, Lee MH, Park JH, Kang SH, Yoo HM, Ka SH, Oh YM, Jeon YJ and Chung CH: SUMOylation of hnRNP-K is required for p53-mediated cell-cycle arrest in response to DNA damage. EMBO J 31: 4441-4452, 2012.

36. Lin J, Chen J, Elenbaas B and Levine AJ: Several hydrophobic amino acids in the p53 amino-terminal domain are required for transcriptional activation, binding to $\mathrm{mdm}-2$ and the adenovirus 5 E1B 55-kD protein. Genes Dev 8: 1235-1246, 1994.

37. Poyurovsky MV, Katz C, Laptenko O, Beckerman R, Lokshin M, Ahn J, Byeon IJ, Gabizon R, Mattia M, Zupnick A, Brown LM, Friedler A and Prives C: The C-terminus of $\mathrm{p} 53$ binds the N-terminal domain of MDM2. Nat Struct Mol Biol 17: 982-989, 2010. 